Proyecciones Journal of Mathematics

Vol. 34, No 3, pp. 243-254, September 2015.

Universidad Católica del Norte

Antofagasta - Chile

\title{
Skolem Difference Mean Graphs
}

\author{
M. Selvi \\ D. Ramya \\ Dr. Sivanthi Aditanar College of Engineering, India \\ and \\ P. Jeyanthi \\ Govindammal Aditanar College for Women, India \\ Received : April 2015. Accepted : June 2015
}

\begin{abstract}
A graph $G=(V, E)$ with $p$ vertices and $q$ edges is said to have skolem difference mean labeling if it is possible to label the vertices $x \in V$ with distinct elements $f(x)$ from $1,2,3, \cdots, p+q$ in such a way that for each edge $e=u v$, let $f^{*}(e)=\left\lceil\frac{|f(u)-f(v)|}{2}\right\rceil$ and the resulting labels of the edges are distinct and are from $1,2,3, \cdots, q$. A graph that admits a skolem difference mean labeling is called a skolem difference mean graph. In this paper, we prove $C_{n} @ P_{m}(n \geq 3, m \geq 1)$, $T\left\langle K_{1, n_{1}}: K_{1, n_{2}}: \cdots: K_{1, n_{m}}\right\rangle, T\left\langle K_{1, n_{1}} \circ K_{1, n_{2}} \circ \circ \circ K_{1, n_{m}}\right\rangle$, $\operatorname{St}\left(n_{1}, n_{2}, \cdots, n_{m}\right)$ and $B t(\underbrace{n, n, \cdots, n}_{m \text { times }})$ are skolem difference mean graphs.
\end{abstract}

Keywords : Mean labeling, skolem difference mean labeling, skolem difference mean graph, extra skolem difference mean labeling.

AMS Subject Classification : $05 C 78$ 


\section{Introduction}

Throughout this paper by a graph we mean a finite, simple and undirected one. The vertex set and the edge set of a graph $G$ are denoted by $V(G)$ and $E(G)$ respectively. Terms and notations not defined here are used in the sense of Harary[1]. A graph labeling is an assignment of integers to the vertices or edges or both, subject to certain conditions. There are several types of labeling. An excellent survey of graph labeling is available in [2]. The concept of mean labeling was introduced by Somasundaram and Ponraj [8]. A graph $G=(V, E)$ with $p$ vertices and $q$ edges is called a mean graph if there is an injective function $f$ that maps $V(G)$ to $\{0,1,2, \cdots, q\}$ such that each edge $u v$ is labeled with $\frac{f(u)+f(v)}{2}$ if $f(u)+f(v)$ is even and $\frac{f(u)+f(v)+1}{2}$ if $f(u)+f(v)$ is odd. Then the resulting edge labels are distinct. The notion of skolem difference mean labeling was due to Murugan and Subramanian [3]. A graph $G=(V, E)$ with $p$ vertices and $q$ edges is said to have skolem difference mean labeling if it is possible to label the vertices $x \in V$ with distinct elements $f(x)$ from $1,2,3, \cdots, p+q$ in such a way that for each edge $e=u v$, let $f^{*}(e)=\left\lceil\frac{|f(u)-f(v)|}{2}\right\rceil$ and the resulting labels of the edges are distinct and are from $1,2,3, \cdots, q$. A graph that admits a skolem difference mean labeling is called a skolem difference mean graph. Further studies on skolem difference mean labeling are available in [4]-[7].

In this paper, we extend the study on skolem difference mean labeling and prove that $C_{n} @ P_{m}(n \geq 3, m \geq 1), T\left\langle K_{1, n_{1}}: K_{1, n_{2}}: K_{1, n_{3}}: \cdots: K_{1, n_{m}}\right\rangle$, $T\left\langle K_{1, n_{1}} \circ K_{1, n_{2}} \circ \circ \circ K_{1, n_{m}}\right\rangle, S t\left(n_{1}, n_{2}, \cdots, n_{m}\right)$ and $B t(\underbrace{n, n, \cdots, n}_{m \text { times }})$ are

skolem difference mean graphs.

$$
m \text { times }
$$

We use the following definitions in the subsequent section.

Definition 1.1. The graph $C_{n} @ P_{m}$ is obtained by identifying one pendant vertex of the path $P_{m}$ with a vertex of the cycle $C_{n}$.

Definition 1.2. The shrub $\operatorname{St}\left(n_{1}, n_{2}, \cdots, n_{m}\right)$ is a graph obtained by connecting a vertex $v_{0}$ to the central vertex of each of $m$ number of stars.

Definition 1.3. The banana tree $B t(\underbrace{n_{1}, n_{2}, \cdots, n_{m}}_{m \text { times }})$ is a graph obtained by connecting a vertex $v_{0}$ to one leaf of each of $m$ number of stars.

Definition 1.4. Let $G=(V, E)$ be a skolem difference mean graph with $p$ vertices and $q$ edges. If one of the skolem difference mean labeling of $G$ 
satisfies the condition that all the labels of the vertices are odd, then we call this skolem difference mean labeling an extra skolem difference mean labeling and call the graph $G$ an extra skolem difference mean graph [7].

An extra skolem difference mean labeling of $P_{6}$ is given in Figure 1

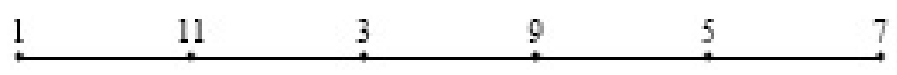

Figure 1

\section{Main Results}

Theorem 2.1. The graph $C_{n} @ P_{m}(n \geq 3, m \geq 1)$ is a skolem difference mean graph.

Proof. Case (i): $n$ is odd.

Let $n=2 k+1$. Let $u_{1}, u_{2}, \cdots, u_{k}, v_{k}, v_{k-1}, \cdots, v_{1}, v_{0}$ be the vertices of $C_{2 k+1}$ and let $w_{1}, w_{2}, \cdots, w_{m}$ be the vertices of $P_{m}$. Then $C_{n} @ P_{m}$ is obtained by identifying $w_{1}$ of $P_{m}$ with $v_{0}$ of $C_{2 k+1}$, which has $n+m-1$ edges. $E\left(C_{2 k+1} @ P_{m}\right)=\left\{v_{i} v_{i+1} \mid 1 \leq i \leq k-1\right\} \cup\left\{u_{i} u_{i+1} \mid 1 \leq i \leq k-1\right\} \cup$ $\left\{w_{j} w_{j+1} \mid 1 \leq j \leq m-1\right\} \cup\left\{v_{0} v_{1}, v_{0} u_{1}, u_{k} v_{k}\right\}$. Define $f: V\left(C_{2 k+1} @ P_{m}\right) \rightarrow$ $\{1,2,3, \cdots, p+q=2 n+2 m-2\}$ as follows:

$f\left(w_{2 i-1}\right)=2 i-1$ for $1 \leq i \leq\left\lceil\frac{m}{2}\right\rceil$,

$f\left(w_{2 i}\right)=2 m+3-2 i$ for $1 \leq i \leq\left\lfloor\frac{m}{2}\right\rfloor$,

$f\left(u_{2 i-1}\right)=2 m+2 n+2-4 i$ for $1 \leq i \leq\left\lceil\frac{k}{2}\right\rceil$,

$f\left(u_{2 i}\right)=4 i$ for $1 \leq i \leq\left\lfloor\frac{k}{2}\right\rfloor$,

$f\left(v_{2 i-1}\right)=2 m+2 n+1-4 i$ for $1 \leq i \leq\left\lceil\frac{k}{2}\right\rceil$,

$f\left(v_{2 i}\right)=4 i+2$ for $1 \leq i \leq\left\lfloor\frac{k}{2}\right\rfloor$.

For each vertex label $f$, the induced edge label $f^{*}$ is calculated as follows:

$f^{*}\left(w_{i} w_{i+1}\right)=m+1-i$ for $1 \leq i \leq m-1$,

$f^{*}\left(u_{k} v_{k}\right)=1$,

$f^{*}\left(u_{i} u_{i+1}\right)=n+m-1-2 i$ for $1 \leq i \leq k-1$, 
$f^{*}\left(v_{i} v_{i+1}\right)=n+m-2-2 i$ for $1 \leq i \leq k-1$, $f^{*}\left(u_{1} v_{0}\right)=n+m-1, f^{*}\left(v_{1} v_{0}\right)=n+m-2$.

Case (ii): $n$ is even.

Let $n=2 k, k>1$. Let $v_{0}, v_{1}, v_{2}, \cdots, v_{k-1}, u_{0}, u_{k-1}, u_{k-2}, \cdots, u_{2}, u_{1}$ be the vertices of $C_{2 k}$ and let $w_{1}, w_{2}, \cdots, w_{m}$ be the vertices of $P_{m}$. Then $C_{n} @ P_{m}$ is obtained by identifying $w_{1}$ of $P_{m}$ with $v_{0}$ of $C_{2 k}$, which has $n+m-1$ edges. $\quad E\left(C_{2 k} @ P_{m}\right)=\left\{v_{i} v_{i+1} \mid 1 \leq i \leq k-2\right\} \cup\left\{u_{i} u_{i+1} \mid 1 \leq i \leq k-2\right\} \cup$ $\left\{w_{j} w_{j+1} \mid 1 \leq j \leq m-1\right\} \cup\left\{v_{0} v_{1}, v_{0} u_{1}, u_{0} u_{k-1}, u_{0} v_{k-1}\right\}$.

Subcase (i): $k>1$ is odd.

Define $f: V\left(C_{2 k} @ P_{m}\right) \rightarrow\{1,2,3, \cdots, p+q=2 n+2 m-2\}$ as follows: $f\left(w_{2 i-1}\right)=2 i-1$ for $1 \leq i \leq\left\lceil\frac{m}{2}\right\rceil$,

$f\left(w_{2 i}\right)=2 m+1-2 i$ for $1 \leq i \leq\left\lfloor\frac{m}{2}\right\rfloor$,

$f\left(u_{2 i-1}\right)=2 m+2 n+2-4 i$ for $1 \leq i \leq \frac{k-1}{2}$,

$f\left(u_{2 i}\right)=4 i$ for $1 \leq i \leq \frac{k-1}{2}$,

$f\left(v_{2 i-1}\right)=2 m+2 n+1-4 i$ for $1 \leq i \leq \frac{k-1}{2}$,

$f\left(v_{2 i}\right)=4 i+2$ for $1 \leq i \leq \frac{k-1}{2}$, and $f\left(u_{0}\right)=2(n+m-k)$.

For each vertex label $f$, the induced edge label $f^{*}$ is calculated as follows:

$f^{*}\left(w_{i} w_{i+1}\right)=m-i$ for $1 \leq i \leq m-1$,

$f^{*}\left(u_{i} u_{i+1}\right)=n+m-1-2 i$ for $1 \leq i \leq k-2$,

$f^{*}\left(v_{i} v_{i+1}\right)=n+m-2-2 i$ for $1 \leq i \leq k-2$,

$f^{*}\left(v_{0} u_{1}\right)=n+m-1$,

$f^{*}\left(v_{0} v_{1}\right)=n+m-2$,

$f^{*}\left(u_{k-1} u_{0}\right)=m+1$,

$f^{*}\left(v_{k-1} u_{0}\right)=m$.

Subcase (ii): $k>1$ is even.

Define $f: V\left(C_{2 k} @ P_{m}\right) \rightarrow\{1,2,3, \cdots, p+q=2 n+2 m-2\}$ as follows:

$f\left(w_{2 i-1}\right)=2 i-1$ for $1 \leq i \leq\left\lceil\frac{m}{2}\right\rceil$,

$f\left(w_{2 i}\right)=2 m+1-2 i$ for $1 \leq i \leq\left\lfloor\frac{m}{2}\right\rfloor$,

$f\left(u_{2 i-1}\right)=2 m+2 n+2-4 i$ for $1 \leq i \leq \frac{k}{2}$,

$f\left(u_{2 i}\right)=4 i$ for $1 \leq i \leq \frac{k-2}{2}$,

$f\left(v_{2 i-1}\right)=2 m+2 n-4 i$ for $1 \leq i \leq \frac{k}{2}$,

$f\left(v_{2 i}\right)=4 i+1$ for $1 \leq i \leq \frac{k-2}{2}$,

and $f\left(u_{0}\right)=2 k$.

For each vertex label $f$, the induced edge label $f^{*}$ is calculated as follows:

$f^{*}\left(w_{i} w_{i+1}\right)=m-i$ for $1 \leq i \leq m-1$,

$f^{*}\left(u_{i} u_{i+1}\right)=n+m-1-2 i$ for $1 \leq i \leq k-2$,

$f^{*}\left(v_{i} v_{i+1}\right)=n+m-2-2 i$ for $1 \leq i \leq k-2$, 
$f^{*}\left(v_{0} u_{1}\right)=n+m-1$,

$f^{*}\left(v_{0} v_{1}\right)=n+m-2$,

$f^{*}\left(u_{k-1} u_{0}\right)=m+1$

$f^{*}\left(v_{k-1} u_{0}\right)=m$.

It can be verified that $f$ is a skolem difference mean labeling. Hence $C_{n} @ P_{m}$ is a skolem difference mean graph.

Skolem difference mean labelings of $C_{7} @ P_{5}$ and $C_{10} @ P_{5}$ are shown in Figure 2.
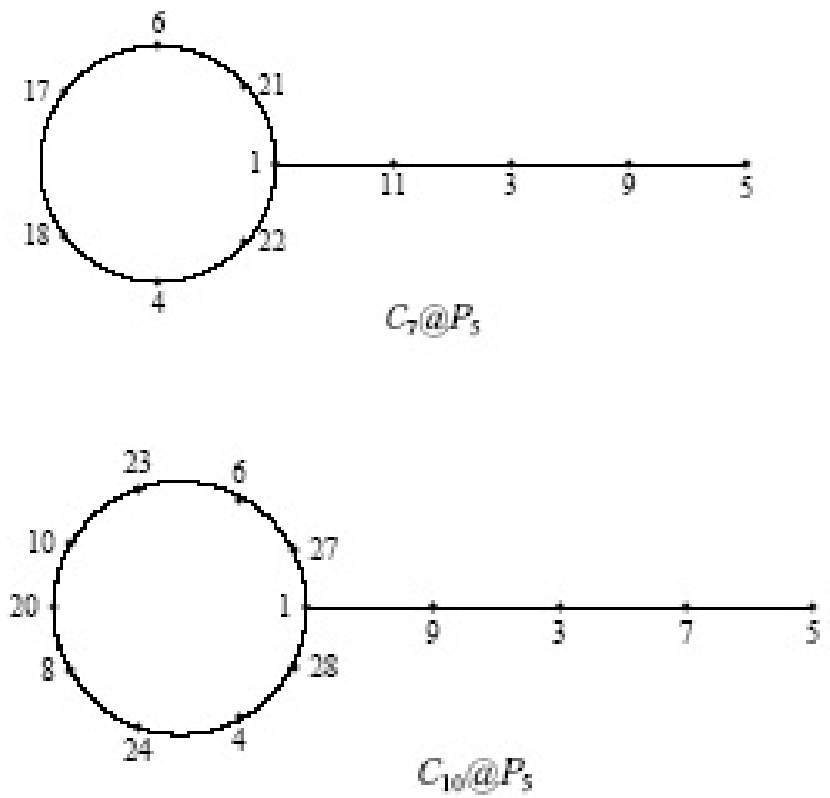

Figure 2

In the following theorem, we prove that the graph $T\left\langle K_{1, n_{1}}: K_{1, n_{2}}: K_{1, n_{3}}: \cdots: K_{1, n_{m}}\right\rangle$, obtained from the stars $K_{1, n_{1}}, K_{1, n_{2}}, \ldots, K_{1, n_{m}}$ by joining the central vertices of $K_{1, n_{j}}$ and $K_{1, n_{j+1}}$ to a new vertex $w_{j}$ for $1 \leq j \leq m-1$ is an extra skolem difference mean graph.

Theorem 2.2. The graph $T\left\langle K_{1, n_{1}}: K_{1, n_{2}}: K_{1, n_{3}}: \cdots: K_{1, n_{m}}\right\rangle$ is an extra skolem difference mean graph.

Proof. Let $u_{i}^{j}\left(1 \leq i \leq n_{j}\right)$ be the pendant vertices and $v_{j}(1 \leq j \leq m)$ be the central vertex of the star $K_{1, n_{j}}(1 \leq j \leq m)$. Then 
$T\left\langle K_{1, n_{1}}: K_{1, n_{2}}: K_{1, n_{3}}: \cdots: K_{1, n_{m}}\right\rangle$ is a graph obtained by joining $v_{j}$ and $v_{j+1}$ to a new vertex $w_{j}(1 \leq j \leq m-1)$ by an edge. Define

$f: V\left(T\left\langle K_{1, n_{1}}: K_{1, n_{2}}: K_{1, n_{3}}: \cdots: K_{1, n_{m}}\right\rangle\right) \rightarrow$

$\left\{1,2,3, \cdots, p+q=2 \sum_{k=1}^{m} n_{k}+4 m-3\right\}$ as follows:

$f\left(v_{j}\right)=2 \sum_{k=1}^{m} n_{k}+4 m-3-2(j-1)$ for $1 \leq j \leq m$,

$f\left(w_{1}\right)=2 n_{1}+1$,

$f\left(w_{j}\right)=2 n_{1}+1+2 \sum_{k=2}^{j}\left(n_{k}+1\right)$ for $2 \leq j \leq m-1$,

$f\left(u_{i}^{1}\right)=2 i-1$ for $1 \leq i \leq n_{1}$

$f\left(u_{i}^{j}\right)=2 \sum_{k=1}^{j-1}\left(n_{k}+1\right)+2 i-1$ for $1 \leq i \leq n_{j}$ and $2 \leq j \leq m$.

For each vertex label $f$, the induced edge label $f^{*}$ is calculated as follows:

Let $e_{i}^{j}\left(1 \leq i \leq n_{j}\right.$ and $\left.1 \leq j \leq m\right)$ be the edges joining the vertices $v_{j}$ with $u_{i}^{j} . f^{*}\left(e_{i}^{1}\right)=\sum_{k=1}^{m} n_{k}+2 m-i-1$ for $1 \leq i \leq n_{1}$,

$f^{*}\left(e_{i}^{j}\right)=n_{j}+n_{j+1}+\cdots+n_{m}+2 m-2 j-i+1$ for $1 \leq i \leq n_{j}$ and $2 \leq j \leq m$, $f^{*}\left(v_{1} w_{1}\right)=n_{2}+n_{3}+\cdots+n_{m}+2(m-1)$,

$f^{*}\left(v_{j} w_{j}\right)=n_{j+1}+n_{j+2}+\cdots+n_{m}+2(m-j)$ for $2 \leq j \leq m-1$, $f^{*}\left(w_{1} v_{2}\right)=n_{2}+n_{3}+\cdots+n_{m}+2 m-3$, $f^{*}\left(w_{j} v_{j+1}\right)=n_{j+1}+n_{j+2}+\cdots+n_{m}+2(m-j)-1$ for $2 \leq j \leq m-1$.

It can be verified that $T\left\langle K_{1, n_{1}}: K_{1, n_{2}}: K_{1, n_{3}}: \cdots: K_{1, n_{m}}\right\rangle$ is an extra skolem difference mean labeling. Hence $T\left\langle K_{1, n_{1}}: K_{1, n_{2}}: K_{1, n_{3}}: \cdots: K_{1, n_{m}}\right\rangle$ is an extra skolem difference mean graph.

Corollary 2.3. The graph $T\langle\underbrace{K_{1, n}: K_{1, n}: K_{1, n}: \cdots: K_{1, n}}_{m \text { times }}\rangle$ is an extra skolem difference mean graph.

An extra skolem difference mean labeling of $T\left\langle K_{1,7}: K_{1,7}: K_{1,7}\right\rangle$ is shown in Figure 3. 


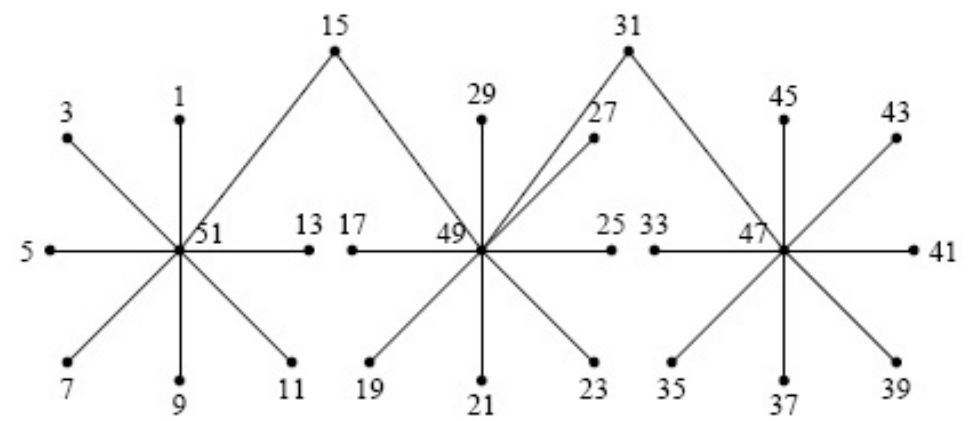

Figure 3

The graph $T\left\langle K_{1, n_{1}} \circ K_{1, n_{2}} \circ \circ \circ K_{1, n_{m}}\right\rangle$ is obtained from the stars $K_{1, n_{1}}, K_{1, n_{2}}, \cdots, K_{1, n_{m}}$ by joining a leaf of $K_{1, n_{j}}$ and a leaf of $K_{1, n_{j+1}}$ to a new vertex $w_{j}(1 \leq j \leq m-1)$ by an edge.

Theorem 2.4. The graph $T\left\langle K_{1, n_{1}} \circ K_{1, n_{2}} \circ K_{1, n_{3}} \circ \circ \circ K_{1, n_{m}}\right\rangle$ is an extra skolem difference mean graph.

Proof. Let $u_{i}^{j}\left(1 \leq i \leq n_{j}\right)$ be the pendant vertices and $v_{j}(1 \leq j \leq m)$ be the central vertex of the star $K_{1, n_{j}}(1 \leq j \leq m)$. Then

$T\left\langle K_{1, n_{1}} \circ K_{1, n_{2}} \circ K_{1, n_{3}} \circ \circ \circ K_{1, n_{m}}\right\rangle$ is a graph obtained by joining $u_{n_{j}}^{j}$ and $u_{1}^{j+1}$ to a new vertex $w_{j}(1 \leq j \leq m-1)$ by an edge. Define

$f: V\left(T\left\langle K_{1, n_{1}} \circ K_{1, n_{2}} \circ K_{1, n_{3}} \circ \circ \circ K_{1, n_{m}}\right\rangle\right) \rightarrow$

$\left\{1,2,3, \cdots, p+q=2 \sum_{k=1}^{m} n_{k}+4 m-3\right\}$ as follows:

$$
f\left(v_{j}\right)=2 \sum_{k=1}^{m} n_{k}+4 m-4 j+1 \text { for } 1 \leq j \leq m,
$$

$f\left(w_{j}\right)=2 \sum_{k=1}^{m} n_{k}+4 m-4 j-1$ for $1 \leq j \leq m-1$,

$f\left(u_{i}^{1}\right)=2 i-1$ for $1 \leq i \leq n_{1}$

$f\left(u_{i}^{j}\right)=2 \sum_{k=1}^{j-1} n_{k}+2 i-1$ for $1 \leq i \leq n_{j}$ and $2 \leq j \leq m$.

Let $e_{i}^{j}=v_{j} u_{i}^{j}$ for $1 \leq i \leq n_{j}$ and $1 \leq j \leq m$. For each vertex label $f$, the induced edge label $f^{*}$ is calculated as follows: $f^{*}\left(e_{i}^{1}\right)=\sum_{k=1}^{m} n_{k}+2 m-i-1$ for $1 \leq i \leq n_{1}$, $f^{*}\left(e_{i}^{j}\right)=n_{j}+n_{j+1}+\cdots+n_{m}+2 m-2 j-i+1$ for $2 \leq j \leq m-1$ and 
$1 \leq i \leq n_{j}$

$f^{*}\left(u_{n_{1}}^{1} w_{1}\right)=\sum_{k=1}^{m} n_{k}+2 m-2-n_{1}$,

$f^{*}\left(u_{n_{j}}^{j} w_{j}\right)=n_{j+1}+n_{j+2}+\cdots+n_{m}+2(m-j)$ for $2 \leq j \leq m-1$,

$f^{*}\left(w_{1} u_{1}^{2}\right)=n_{2}+n_{3}+\cdots+n_{m}+2 m-3$,

$f^{*}\left(w_{j} u_{1}^{j+1}\right)=n_{j+1}+n_{j+2}+\cdots+n_{m}+2 m-2 j-1$ for $2 \leq j \leq m-1$.

It can be verified that $T\left\langle K_{1, n_{1}} \circ K_{1, n_{2}} \circ K_{1, n_{3}} \circ \circ \circ K_{1, n_{m}}\right\rangle$ is an extra skolem difference mean labeling. Hence $T\left\langle K_{1, n_{1}} \circ K_{1, n_{2}} \circ K_{1, n_{3}} \circ \circ \circ K_{1, n_{m}}\right\rangle$ is an extra skolem difference mean graph.

Corollary 2.5. The graph $T\langle\underbrace{K_{1, n} \circ K_{1, n} \circ K_{1, n} \circ \circ \circ K_{1, n}}_{m \text { times }}\rangle$ is a skolem difference mean graph.

A skolem difference mean labeling of $T\left\langle K_{1,6} \circ K_{1,6} \circ K_{1,6} \circ K_{1,6}\right\rangle$ is shown in Figure 4.

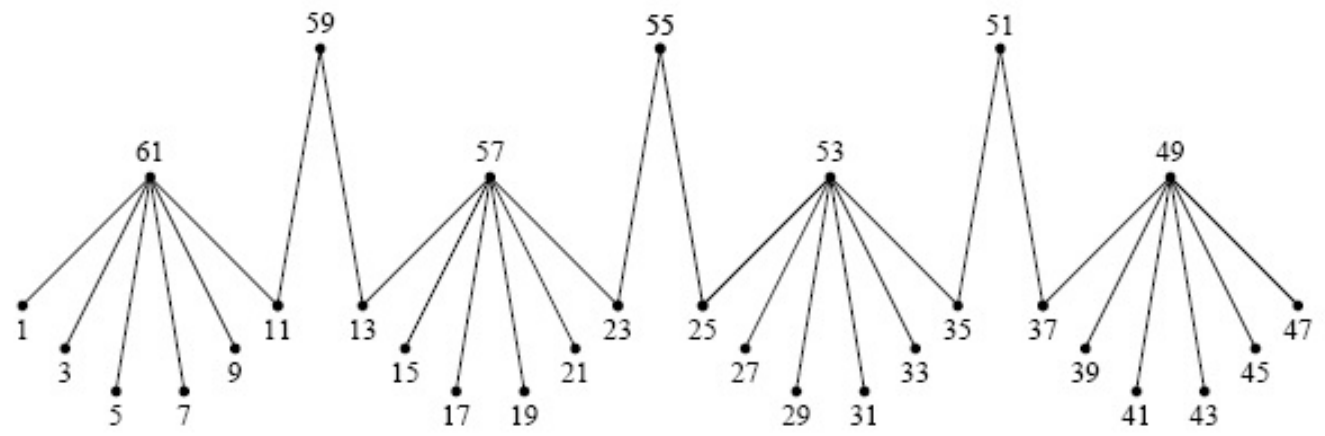

Figure 4 
Theorem 2.6. If $G$ is a graph having $p$ vertices and $q$ edges with $q>p$ then $G$ is not a skolem difference mean graph.

Proof. Let $G$ be a $(p, q)$ graph with $q>p$. The minimum possible vertex label of $G$ is 1 and the maximum possible vertex label of $G$ is $p+q$. Therefore, the maximum possible edge label of $G$ is $\left\lceil\frac{p+q-1}{2}\right\rceil<\left\lceil\frac{2 q-1}{2}\right\rceil=q$. That is, $G$ has no edge having the label $q$ and hence $G$ is not a skolem difference mean graph.

Corollary 2.7. The complete graph $K_{n}$ is a skolem difference mean graph if and only if $n \leq 3$.

Proof. $\quad K_{2}$ is $P_{2}$ and $K_{3}$ is $C_{3}$. The graph $K_{n}$ has $n$ vertices and $\frac{n(n-1)}{2}$ edges. For $n \geq 4, \frac{n(n-1)}{2}>n$. By theorem 2.6, $K_{n}, n \geq 4$ is not a skolem difference mean graph.

Theorem 2.8. The shrub $\operatorname{St}\left(n_{1}, n_{2}, \cdots, n_{m}\right)$ is a skolem difference mean graph.

Proof. Let $v_{0}, v_{j}, u_{i}^{j}\left(1 \leq j \leq m, 1 \leq i \leq n_{j}\right)$ be the vertices of $\operatorname{St}\left(n_{1}, n_{2}, \cdots, n_{m}\right)$. Then

$E\left(S t\left(n_{1}, n_{2}, \cdots, n_{m}\right)\right)=\left\{v_{0} v_{j} \mid 1 \leq j \leq m\right\} \cup\left\{v_{j} u_{i}^{j} \mid 1 \leq i \leq n_{j}\right\}$.

Define $f: V\left(S t\left(n_{1}, n_{2}, \cdots, n_{m}\right)\right) \rightarrow\left\{1,2,3, \cdots, p+q=2 \sum_{j=1}^{m} n_{j}+2 m+1\right\}$

as follows:

$$
f\left(v_{0}\right)=2 m+2 \sum_{j=1}^{m} n_{j}+1,
$$

$f\left(v_{j}\right)=2 j-1,1 \leq j \leq m$,

$f\left(u_{i}^{j}\right)=2\left(n_{j}+n_{j+1}+\cdots+n_{m}\right)+2(j-i), 1 \leq i \leq n_{j}-1$ and $1 \leq j \leq m$, $f\left(u_{n_{j}}^{j}\right)=2\left(n_{j+1}+n_{j+2}+\cdots+n_{m}\right)+2 j+1$ for $1 \leq j \leq m-1$, $f\left(u_{n_{m}}^{m}\right)=2 m+1$.

Let $e_{i}^{j}=v_{j} u_{i}^{j}$ for $1 \leq i \leq n_{j}$ and $1 \leq j \leq m$. For each vertex label $f$, the induced edge label $f^{*}$ is defined as follows: $f^{*}\left(v_{0} v_{j}\right)=m+n_{1}+n_{2}+$ $\cdots+n_{m}-j+1,1 \leq j \leq m$ $f^{*}\left(e_{i}^{j}\right)=n_{j}+n_{j+1}+\cdots+n_{m}-i+1,1 \leq i \leq n_{j}$ and $1 \leq j \leq m$.

It can be verified that $S t\left(n_{1}, n_{2}, \cdots, n_{m}\right)$ is a skolem difference mean graph. 
The skolem difference mean labeling of $S t(2,5,5,3)$ is shown in Figure 5 .

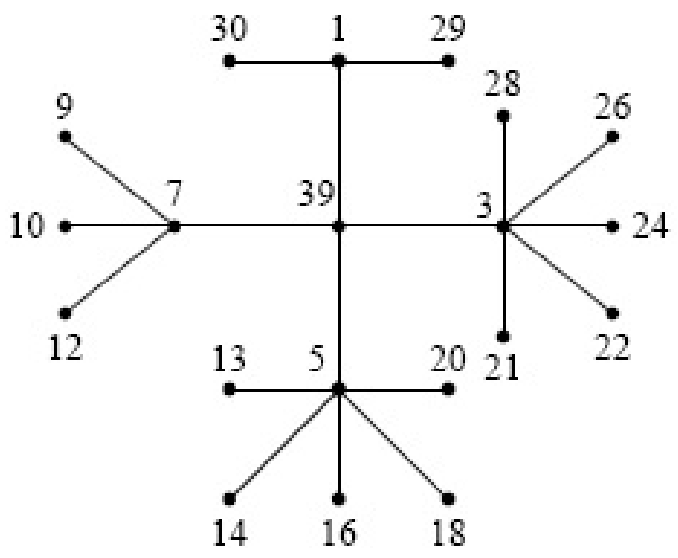

Figure 5

Theorem 2.9. The banana tree $B t(\underbrace{n, n, \cdots, n}_{m \text { times }})$ is a skolem difference mean graph.

Proof. Let $v_{0}, v_{j}, u_{i}^{j}(1 \leq j \leq m, 1 \leq i \leq n)$ be the vertices of $B t(\underbrace{n, n, \cdots, n}_{m \text { times }})$. Then $E(B t(\underbrace{n, n, \cdots, n}_{m \text { times }}))=\left\{v_{0} u_{1}^{j}, v_{j} u_{i}^{j} \mid 1 \leq i \leq n\right.$ and $\left.1 \leq j \leq m\right\}$.

lows:

Define $f: V(B t(\underbrace{n, n, \cdots, n}_{m \text { times }})) \rightarrow\{1,2,3, \cdots, p+q=2 m(n+1)+1\}$ as fol-

$$
f\left(v_{0}\right)=2 m(n+1)+1
$$

$f\left(v_{j}\right)=2 m(n-1)+4 j-1$, for $1 \leq j \leq m$,

$f\left(u_{1}^{j}\right)=2 j-1,1 \leq j \leq m$,

$f\left(u_{i}^{j}\right)=(2 i-2) m+2 j-1$, for $2 \leq i \leq n-1$ and $1 \leq j \leq m$,

$f\left(u_{n}^{j}\right)=2 m(n+1)-2(m-j)$ for $1 \leq j \leq m$. Let $e_{i}^{j}=v_{j} u_{i}^{j}$ for $1 \leq i \leq n$ and $1 \leq j \leq m$. For each vertex label $f$, the induced edge label $f^{*}$ is calculated as follows: $f^{*}\left(v_{0} u_{1}^{j}\right)=m(n+1)-j+1$, for $1 \leq j \leq m$ $f^{*}\left(e_{i}^{j}\right)=m(n-i)+j$, for $1 \leq i \leq n-1$ and $1 \leq j \leq m$, $f^{*}\left(v_{j} u_{n}^{j}\right)=m-j+1$ for $1 \leq j \leq m$. 
It can be verified that $B t(\underbrace{n, n, \cdots}_{n, n, \cdots, n})$ is a skolem difference mean graph. $m$ times

A skolem difference mean labeling of $\mathrm{Bt}(3,3,3,3,3,3,3,3)$ is shown in Figure 6 .

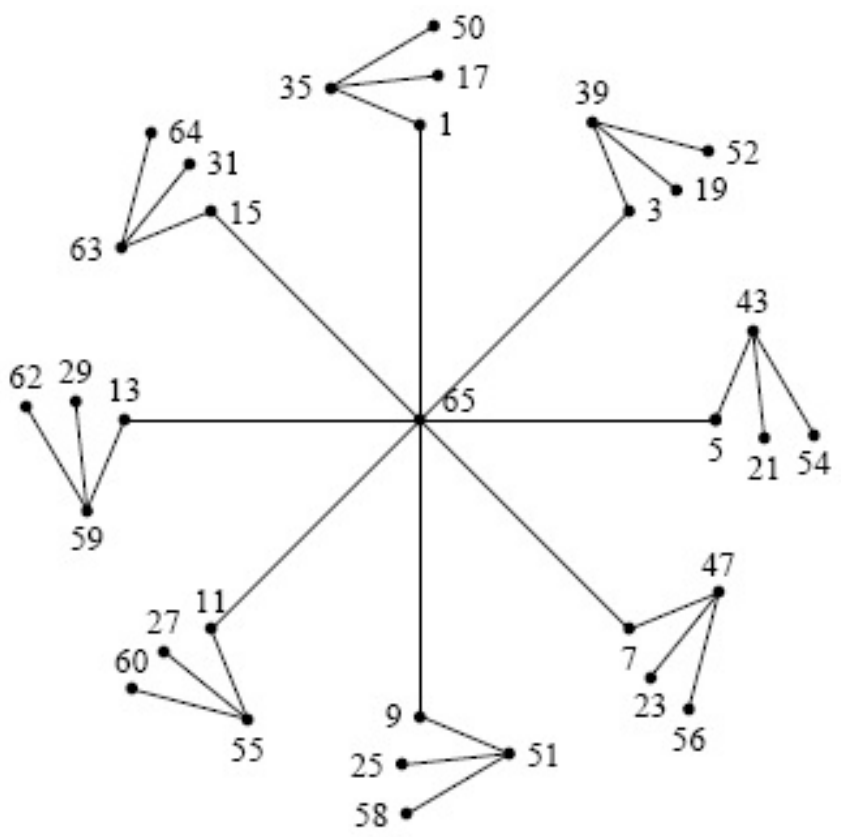

Figure 6

\section{References}

[1] F. Harary, Graph theory, Addison Wesley, Massachusetts, (1972).

[2] Joseph A. Gallian, A Dynamic Survey of Graph Labeling, The Electronic Journal of Combinatorics, 17 (2014), \# DS6.

[3] K. Murugan and A. Subramanian, Skolem difference mean labeling of $H$-graphs, International Journal of Mathematics and Soft Computing, Vol.1, No. 1, pp. 115-129, (2011). 
[4] K. Murugan and A. Subramanian, Labeling of subdivided graphs, American Journal of Mathematics and Sciences, Vol.1, No. 1, pp. 143-149, (2012).

[5] K. Murugan and A. Subramanian, Labeling of finite union of paths, International Journal of Mathematics and Soft Computing, Vol.2, No. 1, pp. 99-108, (2012).

[6] D. Ramya, M. Selvi and R. Kalaiyarasi, On skolem difference mean labeling of graphs, International Journal of Mathematical Archive, 4(12), pp. 73-79, (2013).

[7] D. Ramya and M. Selvi, On skolem difference mean labeling of some trees, International Journal of Mathematics and Soft Computing, Vol.4, No. 2, pp. 11-18, (2014).

[8] S. Somasundaram and R. Ponraj, Mean labelings of graphs, National Academy Science Letter, 26, pp. 210-213, (2003).

\section{Selvi}

Department of Mathematics

Dr. Sivanthi Aditanar College of Engineering, Tiruchendur-628 215, Tamilnadu,

India

e-mail: selvm80@yahoo.in

\section{Ramya}

Department of Mathematics

Dr. Sivanthi Aditanar College of Engineering, Tiruchendur-628 215, Tamilnadu,

India

e-mail : aymar_padma@yahoo.co.in

and

\section{P. Jeyanthi}

Research Centre, Department of Mathematics Govindammal Aditanar College for Women Tiruchendur-628 215, Tamilnadu, India

e-mail : jeyajeyanthi@rediffmail.com 